\title{
La formación investigativa de los estudiantes: Un problema aún por resolver
}

\section{The research training of students: A problem unresolved}

\author{
Narciza Zamora Vera \\ Magister en Educación Superior. Especialista en Diseño Curricular por Competencias. Cursando Doctorado en Ciencias Pedagógicas con la Universidad \\ Oscar Lucero Moya de Cuba. Profesora titular de la Universidad Laica Eloy Alfaro de Manabi (Ecuador) narcizamora@hotmail.es
}

\section{Para citar este artículo:}

Zamora, N. (2014). La formación investigativa de los estudiantes: un problema aún por resolver. Escenarios, 12(2), 76-85.

Recibido: Julio 312014

Aceptado: Septiembre 42014

\begin{abstract}
RESUMEN
El mundo de hoy requiere con urgencia que se encuentren respuestas viables al desarrollo de habilidades investigativas en los estudiantes, a partir de las formas de aprendizajes y los correspondientes métodos, técnicas, lo que supone que el docente desarrolle una enseñanza activa con el auxilio de métodos productivos con miras a cumplir con el encargo social que se plantea a la institución docente. EL enfoque fue dialéctico-materialista, el cual privilegia el paradigma cualitativo de investigación. Y con la observación participante se obtuvo el diagnóstico del estado actual de la formación investigativa en el estudiante y se utilizó también para la evaluación de la estrategia. La formación investigativa es un elemento esencial en la educación, que tiene como objetivo el contribuir a la solución de problemas teóricos, metodológicos y prácticos. Estos se relacionan directamente con el desarrollo científico, inmerso en la rapidez de los cambios que se experimentan en los procesos tecnológicos, organizativos y de la información, lo cual repercute directamente en la economía y otros ámbitos sociales. Se determina que las habilidades científico investigativas son básicas para la formación de los estudiantes y contribuyen a desarrollar competencias para prepáralos en seguir la lógica del proceso de investigación científica. Todo de un modo coherente que conduzca a solucionar un problema científico de evidente connotación social, previamente formulado.
\end{abstract}

Palabras clave: Formación, Investigación, Estudiantes.

\begin{abstract}
Today's world requires urgently viable development of research skills in students from forms of learning and related methods, technical solutions are found, which means that teachers develop active learning methods with the help of production in order to meet the social order that arises in the educational institution. The research training is an essential element in education, with the aim of contributing to the solution of theoretical, methodological and practical problems and is directly related to scientific and technical development, which occurs with the rapid changes experienced in the technological, organizational and information processes, which directly affects the economy and other social areas. Scientific investigative skills are basic to the training of students and help prepare them to continue to develop the logic of scientific research process in a coherent way that leads to solve a scientific problem of obvious social connotation, previously formulated.
\end{abstract}

Key Words: Training, Research, Students. 


\section{Introducción}

El proceso investigativo puede argumentarse desde fundamentos psicopedagógicos, didácticos, psicológicos, lo que se une a la necesidad de aplicar procesos didácticos para estructurary desarrollar las asignaturas de modo que cada una de ellas contribuya al proceso de la investigación formativa.

Los docentes deben tener al menos conocimientos básicos de investigación, e interiorizar la necesidad de fomentar la competencia investigativa desde cada una de las asignaturas que forma parte del pensum de estudio de la unidad académica que permita contribuir en la producción investigativa argumentada desde ideas que desarrollan diversos autores en referencia al tema de los procesos de investigación.

Entre los fundamentos teóricos que sustentan la formación investigativa de los estudiantes de la carrera de Comercio Exterior y Negocios Internacionales se encuentra la concepción socio histórico-cultural de L. S. Vygotsky en relación a la enseñanza, la asistencia pedagógica y el desarrollo psíquico, fortalecida por los puntos de vistas de varios autores y reflexiones propias.

Se debe tener en cuenta que el estudiante universitario debe formarse desde el punto de vista investigativo inmerso en relaciones sociales en correspondencia con el principio vigotskiano de la consideración de lo histórico cultural como determinante del desarrollo psíquico y fuerza motriz de su aprendizaje

Lo antes planteado confirma que la personalidad del estudiante deberá conformarse de forma integral; las cualidades conocimientos y valores aportan a la comprensión de las actividades académicas, laborales e investigativas en las que debe estar implicado el alumno a través del proceso formativo de la carrera.

La concepción vigoskiana sobre las relaciones entre la enseñanza y el desarrollo psíquico, contribuye a orientar metodológicamente el proceso docente-educativo y de manera particular el de formación investigativa del maestro.

\section{Desarrollo para una zona de desarrollo próximo}

En la formación investigativa, desde las posiciones vigotskianas, se concibe que el docente es un mediador del proceso que direcciona sus saberes sobre la base de una actividad interna y externa. El docente, en el transcurso de su proceso pedagógico universitario debe inducir a la reflexión fortaleciendo la comunicación inter y entre docentes y estudiantes a fin de lograr con éxitos su labor educativa. La concreción de acciones en el proceso educativo se logra si el profesor ejecuta acciones con sus estudiantes desde nuevas posiciones teóricas a partir de un proceso de aprendizaje dinámico y participativo.

En la presente tesis se tiene en cuenta que el estudiante debe de apropiarse de conocimientos, pero que estos no son herencia que se traspasa de una persona a otra, si no que van construyendo paso a paso y cada vez con más sólidos y complejos argumentos, a ello se une que las habilidades cognoscitivas se evidencian y se operacionalizan en las acciones sociales.

Los docentes deben preparar al estudiantes para que tomen como referente las necesidades de toda una sociedad y en particular de la región en que se desempeñarán laboralmente a partir de lo que deberán alcanzar el fortalecimiento individual y la internalización, en el paso de lo interpersonal a lo intrapersonal.

El estudiante universitario bajo la guía que el docente le brinda para desarrollar actividades en cada asignatura, debe experimentar cambios significativos en el comportamiento, que en el ámbito lingüístico se evidencien en la instrucción progresiva, en la capacidad de hacer preguntas con mayor significatividad, y sobre todo en la estructuración progresiva del cognitivismo lingüístico puesto de manifiesto en cada acción educativa.

Sin embargo, de lo que se trata es de llevar al estudiante a una zona de desarrollo próximo; contingencias, procesos de instrucción, interrogantes, estructuras de clases, retroalimentación son mecanismos básicos para lograrlo, empleando medios de estructuración cognoscitiva, respuestas a interrogantes instruccio- 
nes en general que posibiliten lograr lo planteado, $y$ permitan que la estructura organizativa se evalúe, y agrupe como una secuencia para la percepción, memoria y la acción, aspectos fundamentales en el proceso investigativo.

\section{Proceso educativo}

Resulta oportuno indicar que el proceso de acuerdo con los razonamientos que se han venido realizando en términos de contextos culturales se hace referencia a la apropiación de conocimientos, al proceso psicológico superior que a través de un proceso educativo se han venido formando.

Que los instrumentos como una mediación semiótica ha jugado un papel fundamental y que la intervención educativa ha dado paso a los procesos psicológicos para fortalecer el lenguaje educativo.

A través de la zona de desarrollo próximo se deberá determinar la capacidad del estudiante para resolver de manera independiente los problemas a un nivel potencial con una guía o experto en el área.

Los docentes en sus actividades educativas deberán tener en cuenta lo sociocultural a fin de contribuir con aportes de la escuela del pensamiento educativos de las diversas disciplinas, dando de esta manera respuesta a las exigencias sociales desde las contribuciones de Vygotsky. Al reconocer el análisis evolutivo, las funciones mentales del estudiante que se derivan de la sociedad y confirmar que el comportamiento educativo se induce por herramientas y signos de forma individual y social, que desde el punto de vista de Marx y Engels, esas herramientas repercuten en la actividad laboral.

En consecuencia el docente en su comunicación con el estudiante debe de aplicar conocimientos psicológicos, en referente a Vygotsky en su concepto de Zona de Desarrollo Próximo lo que debe contribuir al desarrollo humano de manera especial a la formación investigativa.

En consecuencia el cambio del comportamiento educativo del estudiante de Comercio Exterior se evidencia cuando sus percepciones van direccionadas a comprender la necesidad de cambios sociales, cuya conducta individual se caracterice por su grado de desarrollo, que se reconozcan sus capacidades específicas como seres humanos modificando su comportamiento y evidenciando cambios de conducta a través de la enseñanza como un factor indispensable en el proceso de desarrollo educativo.

El docente debe enseñar la investigación de cuestiones que involucran conceptos científicos que se relacionan con los conceptos cotidianos, confrontando al estudiante con la zona de desarrollo próximo mediante tareas docentes de cada una de las asignaturas a fin de guiar su progreso hacia una etapa superior del aprendizaje; estas tareas deberán estar encaminadas a la consecución de métodos para solucionar problemas con la mediación del docente.

En el proceso formativo se deben considerar tres elementos integrados: el educador, el alumno y la situación creada por la interacción. El objetivo es producir un nivel más abstracto de pensamiento con cambios cognoscitivos; con objetivos claros de lo que se persigue de un modo sistemático, que conlleve a formular problemas con tendencia a elevar el nivel cognitivo a partir de las acciones y en consecuencia, hacer mássólida la formación investigativa.

De acuerdo con los razonamientos que se han venido realizando la zona de desarrollo próximo, entendida como la distancia entre la capacidad individual del estudiante y la capacidad para ejecutar con ayuda, requiere del docente vastos conocimientos y capacidad para guiar a los estudiantes de manera correcta a la consecución de objetivos de acuerdo a las asignaturas que imparte y a los logros que desea en el período de clases.

La zona de desarrollo próximo, a partir de los argumentos de Vygotsky se concibe como la distancia entre el nivel real de desarrollo determinado por la resolución de problemas de manera individual y el nivel de desarrollo potencial determinado a través de solución de problemas con la guía del docente o en colaboración con compañeros más capaces (Vigotsky, 1987). 
Es así que los estudiantes con la guía del docente y a través de un proceso investigativo deben aprehender el conocimiento de manera significativa. Las tareas que ayudan a los estudiantes a utilizar el conocimiento de manera significativa incluyen tres características: son tareas a largo plazo, multidimensionales, y dirigidas por el alumno.

\section{Contextualización}

La Filosofía es un elemento que permite comprender y transformar cualquier proceso de actividad humana, es reflexionar acerca de la naturaleza, y la sociedad en general a partir de una relación activa del ser humano con la realidad. La educación y el proceso de enseñanzaaprendizaje, enmarcado en la investigación orientan todo conocimiento que conlleva a formar hábitos, habilidades, actitudes y valores necesarios para que el ser humano logre solucionar problemas e insertarse activa y eficazmente en la sociedad.

En correspondencia de esta forma la relación de los fundamentos filosóficos permite lograr en los estudiantes un aprendizaje significativo y desarrollador sin necesidad de que sean entes reproductivos, sino que por el contrario, deberán estar en condiciones de enfrentar las complejidades que se presenten en la sociedad del presente, a la vez que se preparan para el futuro.

Entre los fundamentos filosóficos que sustentan la formación investigativa de los estudiantes de la carrera de Comercio Exterior y Negocios Internacionales se encuentra la Concepción Materialista de la Historia, cuya esencia es la ley sociológica general que plantea que el ser social determina la conciencia social (Marx, 1975).

El análisis filosófico del proceso formativo y de la formación investigativa de la Facultad de Comercio Exterior y Negocios Internacionales requiere tener en cuenta una serie de aspectos, entre ellos el origen de cada uno de los estudiantes que es muy heterogéneo, con diferentes niveles de cultura que los ubican en determina clases sociales, entre estos estudiantes prevalecen los de clase media baja, lo que se revela en su forma de vida, lugares donde habitan en correspondencia con sus ingresos económicos y nivel educativo, lo que debe ser tenido en cuenta para un enfoque personalizado de la formación investigativa.

Muchos de estos estudiantes residen en sectores rurales, situados a considerable distancia del centro de estudios, motivo para que frecuentemente lleguen al finalizar las horas clases o simplemente a mediados de ella, lo que constituye una de las limitantes para poder integrarse a los trabajos grupales que en algunos casos, se ven obligados a realizar individualmente, y ello repercute negativamente sobre su capacidad para el trabajo grupal.

Es evidente en el comportamiento de los estudiantes el nivel de cultura y educación del entorno familiar ya que se refleja en su comportamiento, seguridad en su actuación y desarrollo intelectual, oportunidad para que el docente aplique las estrategias educativas apropiadas y pueda lograr el cambio en el nivel de desarrollo de las competencias que en algunos casos, están potencialmente presentes en el estudiante, pero que no ha tenido la oportunidad de explorarla.

Generalmente el estudiante se va involucrando y cambiando su comportamiento pero en todo momento necesita del apoyo del docente y es cuando debe tener técnicas y herramientas adecuadas y oportunas para brindar al estudiante la oportunidad de desarrollar esas habilidades internas o intrínsecas en él. Existen estudiantes que por temor a ser juzgados de forma burlesca por sus compañeros se resisten arealizar algún comentario con respecto a la clase y lo que es más a preguntar acerca de algún tema que no fue esclarecido para apropiarse de su contenido.

Han existido casos en que el docente margina a determinados estudiantes, sin darles oportunidad para desplegar sus capacidades, llegan a decirles que deberían de estar realizando actividades agrícolas, porque esas no requieren de conocimientos científicos y educativos y que están perdiendo el tiempo y la oportunidad para dedicarse a otras labores. En algunos casos el estudiante queda influenciado por esos comentarios y termina repitiéndose el mismo que no es capaz de 
llegar a ser un profesional. Esta aseveración repetida muchas veces se va tornando real por lo que a la persona le resulta más difícil demostrar que es capaz de alcanzar las metas trazadas.

La situación antes descrita requiere que el docente emplee sus conocimientos y se apoye en lo mejor de su subjetividad para hacer que este estudiante con un grado bajo de autoestima sea capaz de provocar una transformación positiva que conlleve a desarrollar sus potencialidades como estudiante, aquí no solo está favoreciendo el desarrollo de un ser humano sino a toda una sociedad porque al alcanzar una formación investigativa adecuada, él va a contribuir a su desarrollo.

En consecuencia, a través de la formación investigativa es posible educar y contribuir a realizar al hombre en su auténtica naturaleza, y en la integridad de sus verdaderas posibilidades, haciendo referencia a Martí (1975) Toda Pedagogía supone una visión filosófica del hombre.

Es necesario que los docentes a través de las diversas asignaturas contribuyan a la formación investigativa en cada uno de los estudiantes que a más de los contenidos científicos, también involucre otros aspectos que están relacionados con la vida cotidiana, con sus valores, metas y el rol protagónico que juegan estos aspectos en cada etapa de su vida, de manera que investigadores y estudiantes sientan la necesidad de un cambio educativo, de transformaciones desarrolladoras en correspondencia a las condiciones histórico-concretas.

En el caso antes expuesto, el cambio se concibe como un proceso de mejoramiento educativo, con la finalidad de llegar a la excelencia en correspondencia con las demandas que la sociedad exige.

Es necesario que se alcancen niveles hacia nuevos estadios de desarrollo progresivo, rescatando las potencialidades que cada uno de los estudiantes posee pero que necesitan de la guía del docente para poder desarrollarlas en aras de la formación completa como seres humanos.
Al fortalecer la formación investigativa se puede lograr cambios educativos que se proyecta a la transformación de un proceso de conocimientos que repercute en el entorno social pues a través de un cambio cualitativo por ende va a transformar una sociedad.

De manera general los estudiantes presentan dificultades para apropiarse del conocimiento y sobre todo para desarrollar las habilidades investigativas, lo cual repercute en la planificación del proceso pedagógico y se manifiestan en la repetición de contenidos de forma textual sin análisis ni interpretación de los contenidos; es así que en la mayoría de los casos las actividades que realizan no logran ser aplicadas en la práctica y peor aún, lo aprehendido no pueden ser transferido a nuevas situaciones del entorno social.

El docente debe posibilitar aprendizajes significativos en sus estudiantes de modo que logren concebir soluciones creativas a problemas del medio. La imposición de criterios por parte del profesor no permite que el estudiante enriquezca sus conocimientos y los comparta y en muchos casos limita el análisis crítico que pudiera realizar el grupo, lo que conduce a la discontinuidad de procesos, y a que solo se limiten a realizar actividades para obtener una nota. Las consecuencias expuestas repercuten negativamente sobre el proceso de aprendizaje, se obstaculiza la formación investigativa, baja el nivel académico y la pertinencia integral de la Facultad.

Se pretende que los estudiantes sean transformadores de la sociedad, que contribuyan a solucionar problemas de toda índole desde su propia cultura y sobre todo que se manifieste la capacidad creadora y eleve su autoestima.

Es necesario entonces llevar a cabo un aprendizaje desarrollador que a más de asentarse en fundamentos filosóficos conduzca a integrar conocimientos y ponerlos en práctica en la docencia y a través de la investigación para que lo aprehendido sean útil para la práctica, conduzca al desarrollo, resuelva contradicciones desde posiciones dialécticas, y alcance una dimensión axiológica en el estudiante que se refleja en una valoración objetiva de la realidad con la que el ser humano se vincula. 
Lo antes expuesto, requiere que el docente tenga presente que el estudiante es un ente activo, participativo, para ello se requiere Integrar la comprensión filosófica del conocimiento, la formación de capacidades para investigar los fenómenos sociales que interactúan con la educación, como lo económico, político y social para satisfacer las necesidades del entorno.

En correspondencia con lo antes dicho se impone precisar interrogantes en torno a cómo contribuir a resolver las insuficiencias en la formación investigativa de los estudiantes de modo que los egresados puedan cumplir con más efectividad su función social.

Etimológicamente el término investigación proviene del verbo in (en) vestigar (hallar, seguir vestigios); es un proceso reflexivo, sistemático, controlado y crítico que tiene por finalidad descubrir o interpretar hechos, fenómenos relaciones y leyes de un determinado ámbito de la realidad. La investigación constituye una búsqueda de hechos un camino para conocer la realidad (Sánchez, J).

La investigación puede ser vista también como una actividad concreta desarrollada por grupos de personas especializadas que generalmente se agrupan en instituciones en la búsqueda de aportes que desde la ciencia, contribuyan a solucionar problemas sociales, o lo que es lo mismo, contribuyan a satisfacer demandas formuladas por la sociedad.

La investigación científica y el método científico se diferencian en que la investigación es un conjunto de fases en la búsqueda de respuestas a una situación problemática y el método es el camino a seguir en la indagación de procedimientos a utilizar a través de las normas y reglas genéricas de actuación científica; en síntesis, la investigación es la actividad de búsqueda y el método son los procedimientos a seguir para el camino de esa búsqueda.

Para lograr un grado mayor de precisión respecto a la problemática que se analiza se realizará un breve análisis crítico de la definición de ciencia.

Según Álvarez (1999), la ciencia se define como el sistema de conocimientos que se adquiere como resul- tado del proceso de investigación científica acerca de la naturaleza, la sociedad y el pensamiento; que está históricamente condicionado en su desarrollo y tiene como base la práctica histórico-social de la humanidad.

Autores como Kedrov (citado por Machado, 1988) acerca de que la ciencia es un sistema de conocimientos y conceptos que expresan resultados del conocimiento de la realidad por el hombre.

Según Juárez (2001) en su tesis doctoral menciona que la formación científico investigativa expresa el desarrollo de un conjunto de capacidades y habilidades profesionales, del pensamiento crítico reflexivo de alto nivel, el desempeño de actitudes creadoras, permiten al estudiante detectar, enfrentar y buscar vías de solución a problemas educativos en el proceso de investigación.

En el ámbito del desarrollo de habilidades investigativas, aparecen nuevas necesidades que conllevan a elaborar alternativas con miras a dar solución a problemas globales que atañen al ser humano; dichas habilidades se pueden conformar en el curso del proceso formativo y conllevan a formular mayores exigencias y a la búsqueda de respuestas más expeditas en el ámbito económico, político y social.

Si se toma como referencia el análisis de Andreiev (1978),quien plantea que el progreso científico social futuro estará determinado en gran medida por el grado de preparación que tendrá el estudiante actual, es decir, el especialista joven del mañana, para abordar con espíritu investigativo y creador, la solución de las tareas prácticas e intelectuales, la investigación entonces debe concebirse desde la sistematicidad que fundamenta e introduce al estudiante en la lógica científica y que a través de los contenidos contribuya a formar el estilo del pensamiento científico.

Del conocimiento que tengan los egresados al abandonar las aulas, depende la capacidad para detectar contradicciones existentes en su esfera de actuación que le permitan enfrentar y resolver problemas profesionales; del bagaje intelectual con que egresen depende en gran medida la capacidad para enfrentar nuevos desafíos. 
El estudiante debe contar con herramientas de aprendizaje necesarias, incorporadas por el docente en su labor educativa que contribuyan a la búsqueda científica y la solución de problemas profesionales, que le permita cumplir exitosamente con la profesionalización de su actividad con la finalidad a una práctica social especializada.

De lo antes expuesto se infiere que el docente debe enseñar a investigar a los estudiantes para lograr un profesional con pleno dominio de conocimientos, hábitos, habilidades, valores y capacidad en su área de especialidad; debe además dirigir el procesos formativo a partir de diagnósticos, formar grupos y revelarles cómo trabajar en equipo, de manera que desarrollen su capacidad para solucionar problemas de la práctica profesional a partir de la investigación científica.

Además el conocimiento científico busca las regularidades, intenta describir la realidad para comprenderlas en sus relaciones y emplea métodos como la reflexión, la observación y la experimentación de los fenómenos. La investigación genera productos socioculturales, que en contextos sociales específicos y en procesos históricos determinados cumplen funciones sociales específicas.

El producto de la investigación científica es el conocimiento científico y a través de las ciencias se asciende al nivel teórico, se plantean problemas de profunda connotación social que de un modo u otro adquieren significación práctica.

La investigación básica que se desarrolla desde la ciencia pura y genera nuevos problemas teóricos, la investigación aplicada que se origina desde una ciencia aplicada y genera una actividad teórica, permite la aplicación del conocimiento científico y la investigación tecnológica que conducen al perfeccionamiento de la tecnología, a la adaptación de teorías científicas para determinados fines y sus resultados influyen en la sociedad.

Álvarez \& Sierra (1999), considera que el proceso de formación científico investigativa es formativo, sistémico y profesional, con una concepción científica, pedagógica, estructurado para prepararlo científicamente a través del trabajo en beneficio de la sociedad.

Como objetivo general se pretende estimular y desarrollar la investigación formativa en los estudiantes universitarios a través del docente y como objetivo específicos: propiciar en el estudiante una conciencia investigativa; fortalecer las debilidades de los estudiantes con relación a la interpretación de lectura; aumentar las actividades de los estudiantes con datos cualitativos y cuantitativos durante el proceso.

Con respecto a los fundamentos teóricos, pensadores como Vigotsky considera que la motivación juega un papel muy importante "es lo que este tiene para aprender significativamente y de la habilidad del docente para despertar e incrementar esa motivación" Para Ausubel, "en el aprendizaje significativo el estudiante logra relacionar la nueva tarea de aprendizaje, en forma racional y no arbitraria con sus conocimientos y experiencias previas, acumuladas en su estructura cognoscitiva". Bruner considera que, "Cuando un estudiante conoce el significado de los conceptos, puede formar frase que contengan dos o más conceptos en las que se afirme o niegue algo".

\section{Metodología}

El proyecto investigativo con enfoque dialécticomaterialista privilegia el paradigma cualitativo de investigación, pero incluye elementos de lo cuantitativo. Fue necesario considerar aspectos relevantes de la investigación empírica, en donde se aplica análisis estadísticos y a través de los resultados se construye nuevos conocimientos. Los métodos, técnicas e instrumentos empíricos fundamentales que se utilizaron en la investigación:

Observación participante: para el diagnóstico del estado actual de la formación investigativa en el estudiante y en la evaluación de la estrategia.

Entrevista en profundidad: a estudiantes del noveno y décimo semestre de la carrera para conocer sus necesidades formativas y las carencias existentes en la formación investigativa, así como a docentes que 
fortalezcan las potencialidades del proceso formativo de los estudiantes de la carrera de Comercio Exterior y Negocios Internacionales para perfeccionar la formación investigativa

Encuesta: a docentes para caracterizar el trabajo en las materias relacionadas con la metodología; a directivos de empresas exportadoras e importadoras para conocer sobre el perfil profesional de los egresados y a los estudiantes desde el primer semestre a octavo semestre de la carrera.

Análisis de documentos: normativos de la carrera Comercio Exterior y Negocios Internacionales para conocer el profesional que se necesita y la concepción de la formación investigativa; del trabajo metodológico en la carrera.

Muestreo opinático: del quinto año de la carrera Comercio Exterior y Negocios Internacionales y el claustro de docentes del mismo para la introducción en la práctica de la estrategia propuesta. Este tipo de muestreo se caracteriza por un esfuerzo deliberado de obtener muestras "representativas" mediante la inclusión en la muestra de grupos típicos.

\section{Resultados y Análisis con una estrategia com- puesta de las etapas con sus correspondientes acciones:}

Los métodos, técnicas e instrumentos empíricos fundamentales que se utilizaron en la investigación y que fueron expuestos en el punto anterior revelaron que:

- No todos los docentes universitarios cuentan con técnicas y herramientas de investigación, aunquelas conocen, están muy lejos de tener las habilidades y preparación adecuadas para ponerlas en práctica.

- La mayoría de los docentes considera que se puede iniciar un proceso de aprendizaje investigativo a través de las diversas asignaturas.

- La mayoría reconoce la necesidad de planificar entre las actividades académicas talleres, seminarios y cursos para la preparación investigativa de los docentes.
- Se constata la necesidad de que el docente en cada una de sus actividades académicas enseñe al estudiante a como investigar utilizando técnicas y herramientas pertinentes.

La información obtenida corroboró la necesidad de concebir una estrategia estimular y desarrollar la investigación formativa en los estudiantes universitarios y además ofreció a la autora del presente trabajo argumentos que la condujeron a coincidir plenamente con la aseveración de Álvarez \& Sierra (1999), en tanto se corresponde plenamente con lo que se ha propuesto al elaborar el presente artículo.

La formación profesional del estudiante se desarrolla con la interacción de los componentes académico, laboral e investigativo, en un proceso formativo para el trabajo científico donde el docente emplea métodos científicos para investigar y solucionar problemas de su práctica laboral (Blanco, 2001).

El estudiante debe contar con herramientas de aprendizaje necesarias, incorporadas por el docente en su labor educativa que contribuyan a la búsqueda científica y la solución de problemas profesionales, que le permita cumplir exitosamente con la profesionalización de su actividad con la finalidad de llevar a cabo una práctica social especializada.

Los aspectos antes expuestos revelan la necesidad de desplegar estrategias para el desarrollo de habilidades investigativas en el estudiante de la unidad académica Comercio Exterior y Negocios Internacionales, de modo que cumpla con los requisitos necesarios para ser un profesional competitivo y actualizado.

De manera que se reafirma que la formación investigativa de los estudiantes como un problema relacionado con las insuficiencias teórico-metodológicas en la formación investigativa que reducen las posibilidades de que los egresados puedan cumplir con efectividad su función social.

Para contribuir a solucionar el problema formulado, la autora concibió una estrategia compuesta de las etapas con sus correspondientes acciones: 
Etapa I: Diagnóstico

Objetivo: Determinar la situación actual de la investigación de los estudiantes de Comercio Exterior y Negocios Internacionales.

\section{Acción I:}

a) Establecer los instrumentos del diagnóstico.

b) Aplicar los instrumentos del diagnóstico.

c) Análisis y valoración de consultas.

d) Socialización de resultados del diagnóstico.

e) Determinación de los resultados.

La primera etapa debe permitir ampliar el diálogo, analizar los problemas existentes con respecto a la investigación y arribar a un diagnóstico de primeramano quepermita definir un proceso de cambio que repercuta de forma positiva en la sociedad.

Etapa II: Análisis del programa de investigación.

Objetivo: Investigar el cómo contribuir al desarrollo de la investigación de los estudiantes de Comercio Exterior y Negocios Internacionales.

\section{Acción II:}

a) Valorar los contenidos de la investigación.

b) Analizar los contenidos y las potencialidades de la investigación.

c) Introducir temas relacionados a la investigación.

d) Dar a conocer los resultados del diagnóstico.

e) Consolidar los resultados.

En la referida etapa se hace necesaria la recopilación de la información a través de la observación directa en las aulas de clase, a fin de poder detectar las limitantes en el desarrollo de las habilidades investigativas yen consecuencia, dar inicio a una serie de acciones encaminadas a fortalecer el proceso de investigación como uno de los ejes principales del perfil profesional.

Etapa III:Rediseño del Programa.

Objetivo:Diseñar el Programa de Investigación.

\section{Acción III:}

a) Determinar hábitos de lectura de textos relacionados con la metodología de la investigación.

b) Aplicar métodos de lectura.

c) Seleccionar textos de investigación.

d) Determinación de los resultados.

Se pretende elaborar un diseño donde se contemple actividades enfocadas al hábito de la investigación de los estudiantes y cada acción debe contribuir a fortalecer desde una base la investigación, con una visión de compromiso académico por parte de los docentes direccionados a cumplir con más efectividad y a través de los estudiantes su función social

Etapa IV: Socialización de actividades.

Objetivo: Socializar las acciones con las autoridades competentes dela carrera de Comercio Exterior y Negocios Internacionales.

\section{Acción IV:}

a) Socializar el planteamiento de la actividad.

b) Realizar la valoración crítica para la probable aceptación.

c) Difundir y compartir con los docentes de la carrera.

d) Planeación de las actividades con períodos establecidos

Es así que la formación investigativa debe revelarse como un proceso sistemático en que cada una de las actividades que debe enfocarse hacia objetivos investigativos. Con el trabajo conjunto se debe lograr consolidar esta actividad como un eje principal de las actividades académicas y obtendrá el cambio de conducta a través del conocimiento a los estudiantes cuyo resultado final es el campo laboral y por ende la sociedad en general.

\section{Conclusiones}

Existe una estrecha relación entre el desarrollo de las ciencias y las demandas sociales, de manera tal que el planteamiento de exigencias sociales constituye un 
estímulo para que las ciencias se desarrollen. En última instancia las necesidades dela producción se convierten en elemento dinamizador para el desarrollo delas ciencias.

La formación investigativa de los estudiantes constituye un problema social de la ciencia que se podría formular en los términos de cómo contribuir a resolver las insuficiencias teórico metodológico en la formación investigativa de los estudiantes de modo que los egresados puedan cumplir con más efectividad su función social.

Una estrategia flexible, dinámica y contextualizada deberá contribuir a resolver las insuficiencias teórico metodológico en la formación investigativa de los estudiantes, de manera que la institución docente cumpla con su encargo social y egrese profesionales preparados para transformar su entorno, transformarse a sí mismo e impulsar a su país hacia niveles superiores de desarrollo.

Corresponde a la universidad preparar a los sujetos que deben contribuir a realizar las transformaciones sociales que deberán impulsar el desarrollo del país; por tanto urge que los docentes desde las diversas asignaturas contribuyan a la formación investigativa de los estudiantes. No hacerlo significaría hipotecar el futuro de la patria.

\section{Referencias}

Andreiev, V. (1978). Evaluación pedagógica de las habilidades investigativas de los alumnos de los grados superiores y de los estudiantes en las condiciones de la programación heurística de la enseñanza. Revista Educación Superior Contemporánea, 1 (21). L Habana.

Álvarez, C. (1999). La escuela en la vida. La Habana. Editorial Pueblo y Educación.

Álvarez, C., \& Sierra, V. (1999) Metodología de la Investigación Científica. Material Digitalizado.

Bane, M.J. (2001). Presidential address. Expertise, advocacy, and deliberation: Lessons from welfare reform. Journal of Policy Analysis and Management, 20(2), 191-197.

Blanco, A. (2001). Introducción a la Sociología de la Educación. La Habana: Ed. Pueblo y Educación.

Brioli, C. (2012) Investigación Educativa, Docencia Universitaria, Volumen XIII, No 1 , Venezuela.
Day, J., \& Kalman, D. (2009). Teaching linear algebra: Issues and resources. The College Mathematics Journal, 32(3), 162-168.

Finn, C., Jr. (2010). Trouble ahead? Philanthropy, 15(3), 36-37. New York

García, G. (2003). Compendio de Pedagogía, Editorial Pueblo y Educación, C. Habana.

Hruz, T. (2000). The costs and benefits of smaller classes in Wisconsin: A further evaluation of the SAGE program. Wisconsin Policy Research Institute Report, 13(6).

Machado, J. (1988). Cómo se forma un Investigador, La Habana: Editorial de Ciencias Sociales.

Marx, K. (1975). Prólogo a la contribución a la crítica de la economía política. Editorial Progreso Moscú.

Martí, J. (1963). Reforma esencial en el programa de las universidades. La Habana. Editorial Nacional de Cuba. MINED (2005): VI Seminario Nacional para Educadores, Editorial Pueblo y Educación, C. Habana.

Miller, D.W. (2001, July 13). The problem with studying vouchers. The Chronicle of Higher Education, pp. A14-A15.

Newton-Smith, W.H. (2000). A companion to the philosophy of science. Malden, MA: Blackwell.

Nocedo, I. et al (2002): Metodología de la Investigación Educacional (segunda parte), Editorial Pueblo y Educación. C. Habana.

Pérez, G. et al (2002): Metodología de la Investigación Educacional (primera parte), Editorial Pueblo y Educación. C. Habana.

Pindado, J. (2004) Guía práctica para docentes. Madrid. CEDRO

Sánchez, J. "Problemas sociales de la ciencia y la tecnología” Material Básico. Universidad de Holguín, Cuba.

Sevillano, M. L. (2007) La evaluación de los procesos y productos tecnológico-didácticos. En Ortega, J. A. Chacon, A. (Coord) Nuevas Tecnologías para la Educación en la Era Digital. Madrid. Pirámide. Pp. 367-385

Thiesen, J. (2001). Potencialidades del currículo de pedagogía en la formación científico investigativa de sus estudiantes. Tesis Doctoral. Universidad do Oeste de Santa Catarina: UNOESC.

Vigostky, L.S. (1987): Historia del desarrollo de las funciones psíquicas superiores. La Habana. Editorial Científico Técnica. 